\title{
Algorithms for managing third stage abnormalities
}

\author{
Jason Marcus ${ }^{1}$ and Sue Fawcus ${ }^{1}$ \\ ${ }^{1}$ University of Cape Town Faculty of Health Sciences
}

June 15, 2020

\begin{abstract}
The development of clear approaches to the assessment, resuscitation, treatment and monitoring of the four case scenarios are presented as algorithms, based on available evidence. They need to be field tested, and adapted for Artificial intelligence and different settings. Further research is needed around multimodal sequential care package of care for PPH, conservative surgical measures, resuscitation in LMICs, and the psychological impact of PPH on women.
\end{abstract}

\section{Methods}

We developed algorithms for four case scenarios, based on findings of a rapid review on third stage abnormalities, identification of the most common deviations, severity of related complications or clinical outcomes, and discussion with the WHO technical working group. These were as follows: Approach to Postpartum haemorrhage (PPH) after vaginal delivery, Diagnosis and management of uterine atony, Diagnosis and management of genital tract trauma, Diagnosis and management of retained placenta. A detailed description of the methodology used for the development of the algorithms is provided in Chapter 2

Search strategies The search was directed at research databases (Cochrane, Medline and CINAHL) for evidence based information on the identification and management of third stage abnormalities in low-resource settings, with a focus on PPH. In addition, international and national guidance documents were identified and reviewed (FIGO, RCOG, NICE, ACOG, SA-NCCEMD, ANZCOG, SOGC). Published systematic reviews were also searched and identified. The search was limited to English language literature. Where high quality evidence was lacking, individual trials and some case series were examined.

The literature review was guided by the hierarchy of evidence and prioritised WHO guidelines followed by other international or national guidelines using GRADE methodology. In the absence of guidelines on a case scenario, a combination of existing studies and expert opinion was used to determine key points for consideration in the algorithm. The highest level of evidence found was used to support the decisions along the management pathway, in the order of up-to-date systematic review (with meta-analyses), up-to-date systematic review (without meta-analyses), any available systematic review, validated decision rules, randomized controlled trials, non-randomized controlled trials, observations studies, and consensus documents.

Population and Setting The algorithms were developed to cover the assessment and management of pregnant women with singleton, term pregnancies considered to be low risk, who developed third stage abnormalities after vaginal delivery. Health facilities in low-and middle-income countries were the priority. However, the algorithms are applicable to any health care setting, and possible adaptations that may be required were acknowledged. The target users for these algorithms are skilled health personnel providing care during childbirth working alone or as part of teams, particularly midwives, clinical officers or equivalent, non-specialized clinicians (i.e. clinicians without specialist training in obstetrics but who also provide care for women in labour), and specialists. 
Development of the Algorithms After collating the evidence, a selection process for inclusion of the evidence in the algorithm took place. Selection was based on relevance of the evidence to the key decision points and severity of the condition targeted by an intervention. The selection also accounted for the strength of evidence and applicability and feasibility in a LMIC context. If there were inconsistencies among guidelines, the most up-to-date guidelines and evidence were reviewed and used to inform consultation with experts. A list of inconsistencies was discussed at the WHO Technical Working Group meeting and a consensus reached on evidence for the algorithm. Algorithms were structured to cover criteria that should be used to suspect a deviation from normal third stage of labour, initial assessment, probable causes and potential differential diagnosis, further assessments to reach the most probable diagnosis, and management of the condition. Draw.io, an open source diagramming software, was used to construct the algorithm in a flowchart format. The online software facilitated remote working on SELMA by the WHO Technical Working Group. The algorithm was composed of standardised but variable shaped boxes, representing either a clinical state, decision point, action task, or link to a different algorithm. Each box was numbered and joined to other boxes via arrows, to orientate the reader to the direction of flow. The numbers also corresponded to a table of evidence, showing the evidence source for the action and decision points. They algorithms underwent internal peer review by the WHO Technical Working Group and revisions where needed.

\section{Results}

\section{Search results}

A flow chart of the literature search process and results are shown in the Supplementary material (s1)

(a) International and national guidelines.

$\mathrm{NICE}^{16,43}, \mathrm{FIGO}^{17}, \mathrm{RCOG}^{18}, \mathrm{WHO}^{19}, \mathrm{ACOG}^{20}$ and South African Confidential Enquiry into Maternal Death guidelines (SA-NCCEMD) ${ }^{35}$ guidelines based on evidence and on expert opinion, were all reviewed and shared a common approach: identifying the problem (PPH), calling for assistance, resuscitation and monitoring, identifying the cause of bleeding and initial treatment measures. The RCOG and ACOG guidelines in particular, gave detailed guidance on assessing blood loss and resuscitation measures, including use of blood products.

(b) Research databases and systematic reviews

After exclusions, 13 Cochrane reviews, 19 Medline publications and 9 CINAHL publications were identified. Some of these reviews referred to all four algorithms and some to specific ones.

(c) Other evidence.

The Algorithms for PPH devised by the South African Confidential Enquiry into Maternal Deaths are included. ${ }^{35}$ Relevant articles and research on resuscitation and shock are included. ${ }^{44,45,46}$

Some individual Randomised Controlled trials (e.g. CHAMPION, WOMAN) ${ }^{22,34}$ and some case series related to PPH management are also included. ${ }^{31,32}$

Algorithms were found in the $\mathrm{FIGO}^{17}, \mathrm{RCOG}^{18}$ and SA-NCCEMD ${ }^{35}$ publications; all are consistent with the PPH algorithm being presented.

\section{Four case scenarios for Third stage abnormalities}

Analysis of the evidence and guidance documents on third stage abnormalities enabled the topic to be sub-divided into 4 case scenarios and Algorithms were developed for each. These were as follows:

1. Approach to PPH after vaginal delivery

2. Diagnosis and management of uterine atony

3. Diagnosis and management of genital tract trauma

4. Diagnosis and management of retained placenta 
Each of the four algorithms are led into from the management of third stage of labour algorithm in chapter 3. In addition, algorithm one links to two, three and four.

The themes of (i) maternal assessment of the third stage condition and haemodynamic stability reflected by vital signs (ii) resuscitation and (iii) treatment of the condition, are the basis of all four Algorithms.

The four algorithms are shown in Figures 1 to 4, accompanied by a brief description with reference sources. More detailed evidence summaries for the action boxes in each algorithm can be found in the Supplementary materials (s2).

\section{Algorithm 1: Approach to PPH after vaginal delivery (Figure 1).}

Thirteen Cochrane reviews were identified that dealt with management of PPH: the Mousa review (2014) ${ }^{14}$ was most relevant for an overall approach to $\mathrm{PPH}$, three referred to uterine atony ${ }^{14,21,33}$, five for genital tract trauma ${ }^{48,49,50,51,53}$ and five for retained placenta. ${ }^{36,37,38,39,41}$ The ten trials in the Mousa review dealt with medical management of PPH and acknowledged the lack of evidence for the sequence of interventions and surgical measures. The Mousa review did not cover resuscitation. International and national guidance documents were mostly used to develop the directives for monitoring, resuscitation and treatment sequences. ${ }^{16,17,18,19,20,35,43,46}$

The most frequently used definition of PPH after vaginal delivery is loss of $500 \mathrm{mls}$ blood or more. This appears in the Mousa review and is corroborated in the Meher review ${ }^{15}$ on core outcomes. However, given inaccuracies in routine estimation of blood loss, subjective indicators of blood loss ('brisk bleeding') and deterioration of vital signs ( $\mathrm{SBP}<100 \mathrm{mmHg}$ and Pulse $>110 \mathrm{bpm}$ ) are also described for identifying PPH. Due to the urgency of PPH management, maternal assessment of haemodynamic status, initial resuscitation and immediate measures to stop the bleeding must occur simultaneously (box 2) and be repeated with increased intensification until the problem is resolved. Emphasis is placed on diagnosing the cause of the bleeding. A diagnosis of retained placenta links to Algorithm 4. Current evidence indicates that initial treatment for PPH should include uterine massage, oxytocin and tranexamic acid. Refractory PPH involves further maternal assessment and examination for persistent uterine atony, genital tract tears, retained placental fragments, and less commonly coagulopathy from amniotic fluid embolism and uterine inversion. Initial resuscitation is with crystalloid solutions followed by blood products, the nature of which will depend on whether the hospital has an onsite blood bank or group specific blood in an emergency fridge. Coagulopathy may result from excessive blood loss from any cause. In tertiary centres it can be managed according to point of care or haematology results. In a district hospital management is formulaic relying on either fresh frozen plasma or fresh dried plasma which can be stored on site as a pharmacy item.

\section{Algorithm 2: Uterine atony (Figure 2).}

Three Cochrane reviews were relevant. The Mousa review $(2014)^{14}$; included ten trials which dealt with medical management of PPH and acknowledged the lack of evidence for the sequence of interventions and surgical measures. The other review by Shakur $(2018)^{33}$ was on anti-fibrinolytic drugs. Given the lack of highlevel evidence on the treatment of PPH from uterine atony, an important review on uterotonics for prevention of PPH was considered (Gallos,2018) ${ }^{21}$; it was deemed reasonable to infer efficacy for treatment from efficacy for prevention. As previously described, international and national guidance documents ${ }^{15,16,17,18,19,20,43}$, RCTs on individual treatments ${ }^{22,34}$ and case series were all used to construct this algorithm. Three articles on temporising management for women who need emergency referral which include Non-pneumatic Antishock Garment (NASG), uterine tourniquet and Balloon Tamponade (BT) were also referenced ${ }^{24,29,31,32}$, as well as the Algorithms for PPH devised by the South African Confidential Enquiry into Maternal Deaths. 28,35

Monitoring and resuscitation together with medical measures to arrest bleeding from uterine atony are sequenced in the algorithm. Recommended medications (oxytocin, Syntometrine, misoprostol, Carboprost and TXA) are given and alternatives indicated in the situation of specific contraindications, lack of cold chain and stock-outs of medications. ${ }^{16,17,18,19,20,21,22,23,35,43}$ Heat stable carbetocin was not included as treatment, 
because most of the evidence to date on its use is for prevention. ${ }^{34}$

For surgical interventions, laparotomy with uterine compression sutures, balloon tamponade and uterine artery ligation can be performed with appropriately trained non-specialist doctors at district hospital level; with hysterectomy being a last resort where the skill is available. However, the evidence for the timing and nature of surgical interventions is mostly from expert opinion or based on case series which are included as the evidence. ${ }^{24,25,26,27,28}$ Most evidence for success of balloon tamponade, which is widely used, is based on case reports but two recent RCTs suggest lack of efficacy and increased risk of adverse outcomes. ${ }^{25,30}$ Balloon tamponade is included in the algorithm pending more research with the proviso that it should be a temporising rather than therapeutic measure and abandoned if it fails to control bleeding within 20 minutes. Hysterectomy is a life-saving procedure for intractable uterine atony, but the skill is unlikely to be available at district hospital level where non specialists supervise maternity. ${ }^{27,28,35}$ In this situation, temporising methods (BT, Uterine tourniquet and NASG) $24,31,32$, advice from a specialist and referral to more specialised care are necessary.

\section{Algorithm 3: Genital tract trauma (Figure 3).}

There were 30 Cochrane reviews, involving 185 trials. Most of the trials focused on prevention of perineal tears and management of second stage labour. One was the Mousa 2014 PPH overview ${ }^{14}$, four were on surgical repair $48,49,50,51$, and one on the use of prophylactic antibiotics. ${ }^{53}$

Guidance documents $\left(\mathrm{NICE}{ }^{16,43}, \mathrm{FIGO}^{17}, \mathrm{RCOG}^{18}, \mathrm{WHO}^{19}\right.$, and $\mathrm{ACOG}^{20}$ guidelines) based on evidence and expert opinion, were all reviewed. They all give guidance on the management of perineal tears. There is additional RCOG guidance ${ }^{53}$ on the management of third- and fourth-degree perineal tears and WHO recommendations on use of tranexamic acid. ${ }^{23}$ Guidance on diagnosis and management of uterine rupture was from case reports and expert opinion. ${ }^{27,28,35}$

Genital tract trauma can be identified on routine inspection after vaginal delivery as described in the third stage algorithm or can be actively looked for in cases of refractory PPH not responding to initial management measures. The site of trauma can be perineum (first-, second-, third- and fourth-degree tears), vaginal and cervical tears, vulval and vaginal haematomas, and uterine rupture. Diagnosis of perineal and vaginal tears and haematoma can be made in labour ward, but complex high vaginal and cervical tears may require examination with appropriate analgesia or anaesthesia. Uterine rupture can be diagnosed by uterine exploration and confirmed by laparotomy.

Genital tract trauma associated with PPH requires all the monitoring and resuscitation interventions described in case scenario 1 , with intensification of both when there is haemodynamic instability.

Vaginal, simple cervical, and first- or second-degree perineal tears can be sutured in labour ward with local analgesia. ${ }^{48}$ Complex high vaginal, cervical and third-degree tears require repair with good visualisation for their extent, and appropriate analgesia or anaesthesia in an operating theatre.

Third- and fourth-degree tears are specialised procedures. Cochrane reviews and guidance on the surgical technique indicates no difference between overlapping and end to end techniques. ${ }^{48,} 49,50,51,52,53$ At district hospital, the appropriate skill is often not available, and the patient would need to have vaginal packing to control any bleeding and referral to a specialised centre. There is also evidence for antibiotic cover in these patients. ${ }^{53}$

Suspected uterine rupture requires a laparotomy and may be amenable to surgical repair. ${ }^{27,28,35}$ Extensive ruptures especially with uterine artery involvement need a hysterectomy. The skill for this is not usually available at a district hospital so temporising measures will need to be performed and urgent referral to the next level of care with the appropriate skill. Case series with the uterine tourniquet technique ${ }^{31}$ shows $^{2}$ that it reduces blood loss during transfer, and a cluster randomised controlled trial of the NASG shows a non-significant reduction in recovery from shock. ${ }^{32}$

Algorithm 4. Retained placenta/placental products (Figure 4). 
There were 18 Cochrane reviews, involving 166 trials. Most of the trials focused on AMTSL and prevention of $\mathrm{PPH}$, rather than management. Five were identified that dealt with management of retained placenta. One was the Mousa 2014 PPH overview ${ }^{14}$ which indicates there is minimal evidence on surgical procedures, and the Chongsomchai review which recommends prophylactic antibiotics after manual removal of placenta (MROP) ${ }^{41}$ Three were on treatments found to be ineffective; intraumbilical vein oxytocin ${ }^{36,37}$, nitroglycerine ${ }^{38}$ and prostaglandins. ${ }^{39}$

$\mathrm{NICE}^{6,43}, \mathrm{FIGO}^{17}, \mathrm{RCOG}^{18}, \mathrm{WHO}^{19}, \mathrm{ACOG}^{20}$ and $\mathrm{SA}-\mathrm{NCCEMD}^{35}$ guidelines based on evidence and expert opinion, were all reviewed. NICE provides the definition of prolonged third stage of labour. They all give guidance on the management of retained placenta by manual removal of placenta (MROP) which is a skilled procedure.

Review articles on balloon tamponade are included. ${ }^{24,25,30}$ References for Algorithm 2 are referred to for use of uterotonics and laparotomy procedures.

Retained placenta is diagnosed if it is not expelled within 30 mins of delivery of the baby if AMTSL was employed or one hour for passive management. ${ }^{14,16,17,18,19,20,43}$ It may be detached and lying at the cervical os or in the vagina, it may be still attached, and rarely but more seriously it can be abnormally adherent. The location can be ascertained by vaginal examination or by ultrasound if the skill is available. Despite placental delivery there can be residual placental fragments / products which may cause PPH or uterine sepsis.

The retained placenta algorithm (figure 4) may be entered from the approach to PPH algorithm (figure 1) when bleeding; or the third stage algorithm if it occurs without bleeding

Retained placenta associated with PPH requires all the monitoring and resuscitation interventions described in Algorithm 1, with intensification of both when there is haemodynamic instability.

Management of retained placenta requires removal which can be by repeat controlled cord traction (CCT), or manual removal from the vagina, both of which can be performed in labour ward by a midwife or general doctor. ${ }^{16,17,18,19,29,35,43}$ When the placenta is still attached, a full manual removal of placenta (MROP) is required without delay, preferably in the operating theatre; or in labour ward with adequate analgesia, followed by a five-day course of broad-spectrum antibiotic. ${ }^{41}$

MROP may be followed by PPH from placental site bleeding and uterine atony, the management of which follows the treatment in case scenario 2; with sequential uterotonics and TXA, followed by balloon tamponade. Similarly, laparotomy and further surgical measures are required, as already described for algorithm 2, if medical and BT fails to control the bleeding. $16,17,18,19,20,26,27,28,35,43$

If MROP is complicated due to morbid adherence, it may require uterine curettage under ultrasound, specialist advice and assistance if available; and laparotomy for conservative surgical measures or hysterectomy, if the skill is available.

\section{Discussion}

\section{Main findings}

The algorithm for third stage abnormalities is led into from the Normal third stage management algorithm in chapter 3. It was subdivided into four interlinked algorithms: an approach to PPH after vaginal delivery, uterine atony, genital tract trauma and retained placenta/placental products. Key themes running through each algorithm were maternal assessment, monitoring and resuscitation, and treatment, all of which need to be intensified with worsening haemodynamic status.

\section{Strenghts and limitations}

The strengths of the process were the design of a detailed algorithm with a systematic standardised methodology using the best available evidence, which can be adapted to Artificial Intelligence. Previous algorithms 
(SA, RCOG, FIGO) have broad channels of action lists but do not indicate exactly when and in what circumstances a particular action should be taken.

The challenges and limitations in the algorithm development process includes the lack of evidence for specific components and over-reliance on guidance / expert opinion as sources. In particular, there was a lack of evidence for ordering of interventions and a comprehensive package of care for $\mathrm{PPH}$. These issues were partially resolved by discussions within the SELMA group and peer review.

\section{Interpretation}

It is anticipated that these algorithms will guide practice in managing third stage abnormalities, in particular $\mathrm{PPH}$, and ensure the correct approach is followed and important details are not neglected. Adapting them to mobile phone technology with a built-in advice system from higher levels of care should strengthen the use of the algorithms. Further developments of this process should recognise the need to (a) incorporate referral systems into the algorithms since maternity care in LMICs is organised around levels of care ${ }^{5}$, starting at community based facilities (Primary level care), referral hospitals (Secondary level care) and regional/academic hospitals (Tertiary level care), and (b) consider management of third stage abnormalities following birth at home or in primary care clinics which was not considered in the present work. This will depend on the developement of appropriate content for these settings and the availability of adequate mobile network coverage and device access to care providers. It is important that the algorithms are field tested with feedback from users, to identify problems of interpretation, feasibility and other barriers to impemenation

\section{Conclusion}

This composite PPH algorithm arising from the third stage algorithm, is designed to enable early identification of the 4 case scenarios (PPH after NVD, Uterine atony, Genital tract trauma and Retained placenta/plancental products) and facilitate clear approaches to assessment, monitoring, resuscitation and treatment. There is a need for field testing their applicability in the settings they were developed for, with further content development so that clinical practice may be improved. Further research is needed around the evaluation of a sequential multi modal package of care for PPH, conservative surgical measures, resuscitation in low resource settings and the psychological impact of PPH for women.

\section{Contributions}

WHO conceived the review. JM and SF performed the screening of search outputs, identified eligible studies, and extracted data. SF performed the data analysis, SF and JM developed the algorithms with inputs from the WHO Steering Group. JM and SF wrote the first draft of the paper. All authors contributed to revising the final version and approved the manuscript for publication.

\section{Disclosure of interest}

JM and SF have no conflicts of interest to declare.

\section{References}

1. Carroli G, Cuesta C, Abalos E, Gulmezoglu AM. Epidemiology of postpartum haemorrhage: a systematic review. Best Pract Res Clin Obstet Gynaecol. 2008 Dec;22(6): 999-1012. PubMed PMID: 18819848. Epub 2008/09/25. eng.

2. Say L, Chou D, Gemmill A, Tunçalp Ö, Moller AB, Daniels J, et al. Global causes of maternal death: a WHO systematic analysis. Lancet Glob Health. 2014;2(6):e323-33. PubMed PMID: 25103301. Epub 2014/05/05. eng.

3. Souza JP, Gülmezoglu AM, Vogel J, Carroli G, Lumbiganon P, Qureshi Z, et al. Moving beyond essential interventions for reduction of maternal mortality (the WHO Multicountry Survey on Maternal and Newborn Health): a cross-sectional study. Lancet 2013 ;381(9879):1747-55. PubMed PMID: 23683641. eng.

4. Oyelese Y, Ananth CV. Postpartum hemorrhage: epidemiology, risk factors, and causes. Clin Obstet Gynecol. 2010 Mar;53(1):147-56. PubMed PMID: 20142652. eng. 
5. Fawcus S. Practical approaches to managing postpartum haemorrhage with limited resources. Best Practice and Research in Clinical Obstetrics and Gynaecology 2019; May 7 pii: S1521-6934(19)300434. doi: 10.1016/j.bpobgyn.2019.03.009.

6. Crowe S, Utley M, Costello A, Pagel C. How many births in sub-Saharan Africa and South Asia will not be attended by a skilled birth attendant between 2011 and 2015? BMC Pregnancy Childbirth. 2012 Jan 17;12:4 doi: 10.1186/1471-2393-12-4.

7. Konguny E, Mlava G, Mhango C, van den Broek N. Availability, utilisation and quality of basic

and comprehensive emergency obstetric care services in Malawi. Maternal and Child Health

Journal 2008; 13: 687-94 doi: 10.1186/1471-2393-12-4. DOI 10.1007/s10995-008-0380-y

1. Grady K, Ameh C, Adegoke A, Kongnyuy E, Dornan J, Falconer T, Islam M \& Van den Broek N (2011) Improving essential obstetric and newborn care in resource-poor countries, Journal of Obstetrics and Gynaecology, 2011, 31:1, 18-23, DOI: 10.3109/01443615.2010.533218

2. Ameh C, Msuya S, Hofman J, Raven J, Mathai M, van den Broek N. Status of Emergency Obstetric Care in Six Developing Countries Five Years before the MDG Targets for Maternal and Newborn Health. PLoS ONE 2012, 7(12): e49938. https://doi.org/10.1371/journal.pone.0049938

3. Ameh CA, Van den Broek N, Making It Happen: Training health-care providers in emergency obstetric and newborn care, Best Practice \& Research Clinical Obstetrics \& Gynaecology, 2015 Volume 29, Issue 8, Pages 1077-1091, ISSN 1521-6934, https://doi.org/10.1016/j.bpobgyn.2015.03.019.

4. Myers T, The Value of Care Algorithms. Pharmacotherapy: The Journal of Human Pharmacology and Drug Therapy, 2006, 26: 181S-192S. doi:10.1592/phco.26.9part2.181S

5. Beam AL, Kohane IS. Translating Artificial Intelligence Into Clinical Care. JAMA . 2016;316(22):23682369. doi: 10.1001/jama.2016.17217

6. Patel VL, Shortliffe EH, Stefanelli M, Szolovits P, Berthold MR, Bellazzi R, Abu-Hanna A The coming of age of artificial intelligence in medicine, Artificial Intelligence in Medicine, Volume 46, Issue 1, 2009, Pages 5-17, ISSN 0933-3657, https://doi.org/10.1016/j.artmed.2008.07.017

7. Mousa HA, Blum J, Abou El Senoun G, Shakur H, Alfirevic Z. Treatment for primary postpartum haemorrhage. Cochrane Database of Systematic Reviews 2014, Issue 2. Art. No.: CD003249.DOI: 10.1002/14651858.CD003249.pub3.

8. Meher S, Cuthbert A, Kirkham JJ, Williamson P, Abalos E, Aflaifel N, et al. Core outcome sets for prevention and treatment of postpartum haemorrhage: an international Delphi consensus study. BJOG. 2018 Jun. PubMed PMID: 29920912. Epub 2018/06/19.

9. National Institute for Clinical Excellence (NICE). Intrapartum care for healthy women and babies. Clinical guidelines Published: 3 December 2014, nice.org.uk/guidance/cg190

10. International Federation of Obstetrics and Gynaecology (FIGO). Safe Motherhood and Newborn Health (SMNH) Committee / International Journal of Gynecology and Obstetrics 117 (2012) 108-118

11. Royal College of Obstetricians and Gynaecologists, UK (RCOG). Prevention and Management of Postpartum Haemorrhage, Green-top guideline No.52, December 2016;

12. World Health Organisation (WHO). WHO recommendations for prevention and treatment of postpartum haemorrhage, 2012, WHO, Geneva

13. American College of Obstetricians and Gynaecologists (ACOG). Postpartum Haemorrhage. ACOG Practice Bulleting no 183. 2017; 130(\$): e169-186

14. Gallos ID, et al. Uterotonic agents for preventing postpartum haemorrhage: a network meta-analysis. Cochrane Database of Systematic Reviews 2018, Issue 4. Art. No.: CD011689. CHAMPION, WHO 2012

15. WOMAN Trial Collaborators. Effect of early tranexamic acid administration on mortality, hysterectomy, and other morbidities in women with post-partum haemorrhage (WOMAN): an international, randomised, double-blind, placebo-controlled trial. Lancet 2017; 389: 2105-16

16. World Health Organisation (WHO). WHO recommendation on tranexamic acid for the treatment of postpartum haemorrhage. Geneva: World Health Organization; 2017

17. Revert M, et al. Intrauterine Balloon Tamponade for Severe Postpartum Hemorrhage, in Obstet 
Gynecol 2018;131:143-9

18. Dumont A, Bodin C, Hounkpatin B, et al. Uterine balloon tamponade as an adjunct to misoprostol for the treatment of uncontrolled postpartum haemorrhage: a randomised controlled trial in Benin and Mali. BMJ Open 2017;7: e016590.

19. Doumouchtsis, S; Papageorghiou, A; Arulkumaran, S. Systematic Review of Conservative Management of Postpartum Hemorrhage: What to Do When Medical Treatment Fails. Obstrical Gynecol Survey 2007; 62(8): 540-547

20. Steer P. The surgical approach to postpartum haemorrhage. The Obstetrician and Gynaecologist 2009; 11(4): 231-238.

21. Fawcus S, Moodley J. (2013). Postpartum haemorrhage associated with caesarean section and caesarean hysterectomy. Best practice \& research. Clinical obstetrics \& gynaecology, 27 2, 233-49.

22. Breen M. Temporary treatment of severe postpartum hemorrhage. Int J Gynaecol Obstet. 2012; 118(3):253-4

23. Anger HA, Dabash R, Durocher J, Hassanein N, Ononge S, Frye LJ, Diop A, Beye SB, Burkhardt G, Darwish E, Ramadan MC, Kayaga J, Charles D, Gaye A, Eckardt M, Winikoff B. The effectiveness and safety of introducing condom-catheter uterine balloon tamponade for postpartum haemorrhage at secondary level hospitals in Uganda, Egypt and Senegal: a stepped wedge, cluster-randomised trial. BJOG 2019;126:1612-1621.

24. Wessels U, Mayat N. Use of a uterine tourniquet as a temporizing measure during transfer of patient with obstetric haemorrhage O\&G Forum 2013; 23(3): 29-31

25. Pileggo-Castro C, et al. Non-pneumatic anti-shock garment for improving maternal survival following severe postpartum haemorrhage: a systematic review, in Reproductive Health (2015) 12:28

26. Shakur H, Beaumont D, Pavord S, Gayet-Ageron A, Ker K, Mousa HA. Antifibrinolytic drugs for treating primary postpartum haemorrhage. Cochrane Database of Systematic Reviews 2018, Issue 2. Art. No.: CD012964. DOI: 10.1002/14651858.CD012964

27. Widmer M, Piaggio G, Nguyen TMH, Osoti A, Owa OO, Misra S, et al. Heat-Stable Carbetocin versus Oxytocin to Prevent Hemorrhage after Vaginal Birth. N Engl J Med. 2018;379(8):743-52. PubMed PMID: 29949473. Epub 2018/06/27.

28. Fawcus S. Alerts for managing postpartum haemorrhage. $S$ Afr Med $J$ 2018;108(12):XXX. DOI:10.7196/SAMJ.2018. v 108i12.13420

29. Nardin JM, Weeks A, Carroli G. Umbilical vein injection for management of retained placenta. Cochrane Database of Systematic Reviews 2011, Issue 5. Art. No.: CD001337.DOI: 10.1002/14651858.CD001337.pub2.

30. Weeks A, Mirembe F, Alfirevic Z. The Release Trial: a randomised controlled trial of umbilical vein oxytocin versus placebo for the treatment of retained placenta. BJOG. 2005 Oct; 112(10):1458.

31. Abdel-Aleem H, Abdel-Aleem MA, Shaaban OM. Nitroglycerin for management of retained placenta. Cochrane Database of Systematic Reviews 2015, Issue 11. Art. No.: CD007708.DOI: 10.1002/14651858.CD007708.pub3.

32. Grillo-Ardila CF, Ruiz-Parra AI, Gaitán HG, Rodriguez-Malagon N. Prostaglandins for management of retained placenta.Cochrane Database of Systematic Reviews 2014, Issue 5. Art. No.: CD010312.DOI: 10.1002/14651858.CD010312.pub2.

33. World Health Organisation (WHO). WHO recommendations for prevention and treatment of maternal peripartum infections. World Health Organization. 2015. ISBN 9789241549363 (NLM classification: WQ 256)

34. Chongsomchai C, Lumbiganon P, Laopaiboon M. Prophylactic antibiotics for manual removal of retained placenta in vaginal birth. Cochrane Database of Systematic Reviews 2014, Issue 10. Art. No.: CD004904. DOI: 10.1002/14651858.CD004904.pub3.

35. WHO Reproductive Health Library. WHO recommendation on the use of uterine tamponade for the treatment of postpartum haemorrhage (September 2012). The WHO Reproductive Health Library; Geneva: World Health Organization.

36. National Institute for Clinical Excellence (NICE). Care in Third stage of labour. NICE pathways. 
Published 29 November 2019 http://pathways.nice.org.uk/pathways/intrapartumcare

37. Friedman A, Campbell M, Kline C, Wiesner S, D'Alton M, Shields L Implementing Obstetric Early Warning Systems AJP Rep. 2018 Apr; 8(2): e79-e84. doi: 10.1055/s-0038- 1641569] PMCID: PMC5910060 PMID: 29686937.

38. Nathan HL, Ayadi AE, Hezelgrave NL, Butrick, E, Miller S et al. Shock index: an effective predictor of postpartum haemorrhage. BJOG 2015; 122: 268-276 doi.org/10.1111/1471-0528.13206

39. Cannon JW, Hemorrhagic Shock in N Engl J Med 2018; 378:370-379

40. World Health Organisation (WH0). WHO recommendations for prevention of PPH. Geneva; 2018. https://www.who.int/reproductivehealth/help-health-workers-prevent-pph/en/

41. Elharmeel SMA, Chaudhary Y, Tan S, Scheermeyer E, Hanafy A, van Driel ML. Surgical repair of spontaneous perineal tears that occur during childbirth versus no intervention. Cochrane Database of Systematic Reviews 2011, Issue 8. Art. No.: CD008534.DOI: 10.1002/14651858.CD008534.pub2.

42. Kettle C, Dowswell T, Ismail KMK. Absorbable suture materials for primary repair of episiotomy and second-degree tears. Cochrane Database of Systematic Reviews 2010, Issue 6. Art. No.: CD000006.DOI: 10.1002/14651858.CD000006.pub2

43. Kettle C, Dowswell T, Ismail KMK. Continuous and interrupted suturing techniques for repair of episiotomy or second-degree tears. Cochrane Database of Systematic Reviews 2012, Issue 11. Art. No.: CD000947.DOI: 10.1002/14651858.CD000947.pub3

44. Fernando RJ, Sultan AH, Kettle C, Thakar R.Methods of repair for obstetric anal sphincter injury. Cochrane Database of Systematic Reviews 2013, Issue 12. Art. No.: CD002866.DOI: 10.1002/14651858.CD002866.pub3.

45. Royal College of Obstetricians and Gynaecologists (RCOG). The Management of Third- and FourthDegree Perineal Tears. Green-top Guideline No. 29 June 2015

46. Buppasiri P, Lumbiganon P, Thinkhamrop J, Thinkhamrop B.Antibiotic prophylaxis for third- and fourth-degree perineal tear during vaginal birth.Cochrane Database of Systematic Reviews 2014, Issue 10. Art. No.: CD005125. DOI: 10.1002/14651858.CD005125.pub4.

\section{Hosted file}

Evidence tables case scenarios 1-4.docx available at https://authorea.com/users/333482/ articles/459704-algorithms-for-managing-third-stage-abnormalities

\section{Hosted file}

(S1) Search results flowchart May 2020.docx available at https://authorea.com/users/333482/ articles/459704-algorithms-for-managing-third-stage-abnormalities

\section{Hosted file}

Third stage abnormalities algorithm figures.docx available at https://authorea.com/users/ 333482/articles/459704-algorithms-for-managing-third-stage-abnormalities 